\title{
Plastic Motion of a Flux-line Lattice Driven by Alternating Current
}

\author{
W. Henderson 1 it, M.J. Higgins ${ }^{2}$ and E.Y. Andrei ${ }^{1}$ \\ ${ }^{1}$ Department of Physics and Astronomy, Rutgers University Piscataway, New Jersey 08855 \\ ${ }^{2}$ NEC Research Institute, 4 Independence Way, Princeton, New Jersey 08540
}

\begin{abstract}
We have measured the response of the flux-line lattice in the low $\mathrm{T}_{c}$ superconductor, $2 \mathrm{H}-\mathrm{NbSe}_{2}$, to finite frequency drives. In a well-defined range of fields, temperatures, and driving amplitudes the system exhibits variety of novel non-linear phenomena. Most strikingly, the flux-lines can move easily in response to currents that are significantly lower than the DC critical current if the direction of the current is reversed periodically while the amplitude, I, is kept fixed, but they do not respond to a current that periodically switches between zero and I, while the direction is kept fixed. Pronounced memory effects associated with these phenomena indicate the presence of dynamically generated structural changes in the flux lattice.
\end{abstract}

PACS numbers:

The physics of the flux-line lattice (FLL) in low $T_{c}$ superconductors is governed by a competition between fluxline interactions, which favor the formation of an ordered hexagonal lattice, and pinning by a random distribution of material defects which favor a disordered FLL. Recent work on dynamical transitions that occur when the FLL is driven by an applied current [1] 3] has shown that depinning is a complex process that can involve tearing of the flux lattice and plastic flow [4 [8]. However, while it was shown that the plastic flow is accompanied by an increase in signal noise and that its low frequency response is anomalous [A], little else is known about the AC response in the plastic flow regime.

In this letter, we report the first observation of a rich variety of effects that occur when the flux lattice is driven by alternating currents with amplitudes below the DC critical current [9, 10], in a region of the phase diagram which was previously associated with plastic flow [4. We find that the flux-lines move easily when they are shaken back and forth by a current that periodically reverses direction while the amplitude, I, is kept fixed, but they do not respond to a current that periodically switches between zero and I, while the direction is kept fixed. We observe pronounced memory effects associated with this effect, which we attribute to dynamically generated changes in flux lattice structure which are frozen in when the drive is shut off. The results appear to be related to plasticity effects which occur in ordinary solids.

In most weak-pinning superconductors, the interplay between interactions and disorder leads to a peak in the critical current, $I_{c}$ which can be observed as a function of both field and temperature [4, 8 11]: increasing $\mathrm{T}$ at fixed $\mathrm{H}$ leads to a sharp rise in $I_{c}$ which sets in at $T=T_{m}(H)$, reaches its maximum value at $T_{p}(H)$, and finally goes to zero at the transition temperature $T_{c}(H)$ (see Fig. 2). Studies of the DC current-voltage relation [4, 阳, and fluxflow noise 12 have revealed that the variation of $I_{c}$ in the $\mathrm{H}-\mathrm{T}$ plane is linked to the existence of three states of the FLL which exhibit distinct dynamic properties. For $T<T_{m}(H)$ (or equivalently for $H<H_{m}(T)$ ), in- teractions dominate and the flux-lines form an ordered lattice that responds elastically when driven by a current, $I>I_{c}$. Above $T_{p}(H)$, the system is in a glassy state where the FLL is highly disordered. Between $T_{m}(H)$ and $T_{p}(H)$, the FLL is in an intermediate state in which it behaves like a soft solid that tears when it is depinned. The flux motion in this state is thought to involve the flow of channels of relatively weakly pinned flux-lines past more strongly pinned neighbors [4, 5].8]. Such flux-line channels were also observed in numerical simulations 13 16]. The term plastic flow is used here to describe motion in which different regions of the flux lattice flow with different velocities.

The results shown here are for a single crystal sample $(3 \times .6 \times .015 \mathrm{~mm})$ of the low $T_{c}$ superconductor $2 \mathrm{H}-$ $\mathrm{NbSe}_{2}$. This sample was grown by vapor transport from a commercial $\mathrm{NbSe}_{2}$ powder containing 200ppm Fe impurities, which lead to a depressed value of the zero-field transition temperature, $T_{c}$ of $5.8 \mathrm{~K}$. The results reported here were also observed on two other samples of this material, one of which was grown from a high purity powder and had a $T_{c}$ of $7.2 \mathrm{~K}$, which is the nominal value for this material. We focus on results for an impure sample where the peak effect is more pronounced. Measurements were done by the standard four lead technique.

Fig. 11 shows the response of the flux lattice to three types of drives at $\mathrm{H}=0.5 \mathrm{~T}$ and $\mathrm{T}=4.59 \mathrm{~K}$. The data were taken with a digital oscilloscope and were averaged over many cycles to improve the signal to noise ratio. For the data in Fig. 17a the flux-lines were shaken back and forth by a current that switched between $-30 \mathrm{~mA}$ and $+30 \mathrm{~mA}$ (bidirectional pulses). The measured voltage, which is proportional to the velocity of the flux-lines (averaged over the entire lattice), is about $30 \%$ of what it would be in the free flux flow limit (i.e. in the complete absence of pinning). When the driving current is switched between 0 and $+30 \mathrm{~mA}$ or 0 and $-30 \mathrm{~mA}$ (unidirectional pulses), the response is essentially zero (Fig. 1, b and 11 c). The tiny voltage spikes that coincide with the current switches are due to pickup between the leads and can be ignored (they 

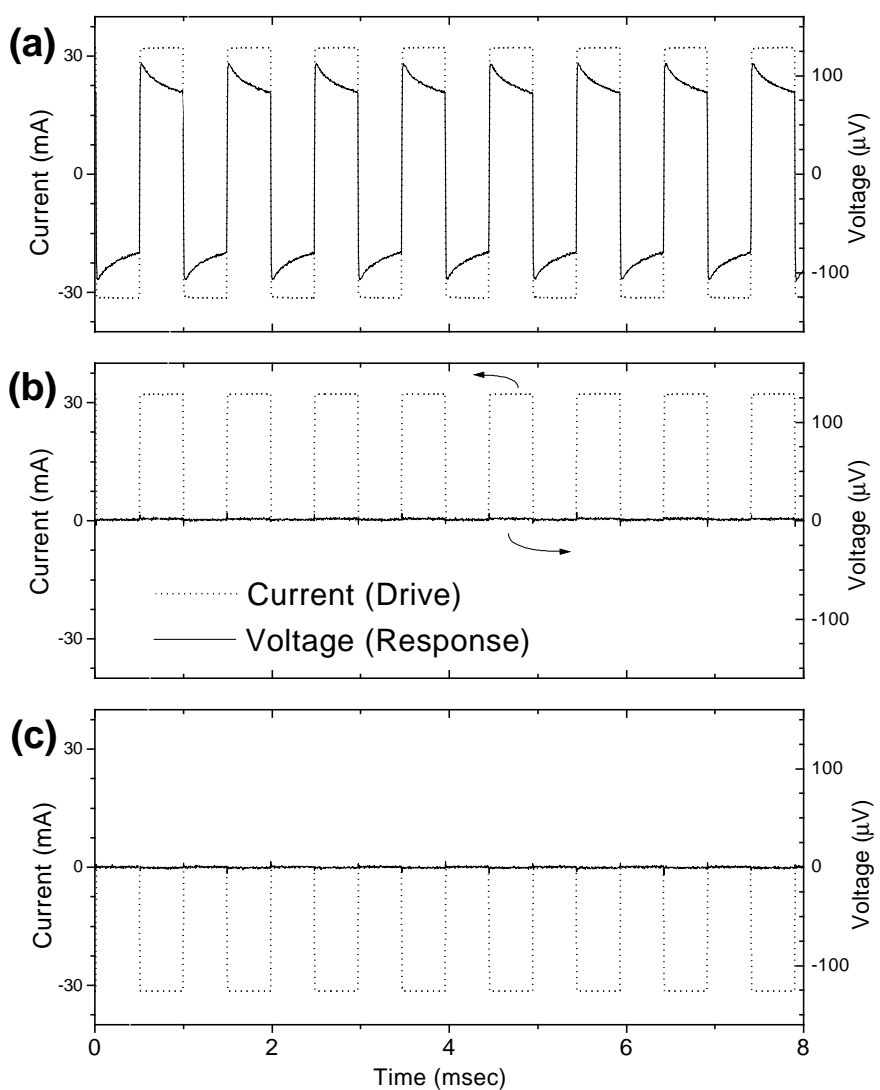

FIG. 1. The response of the flux lattice at $\mathrm{H}=.5 \mathrm{~T}$ and $\mathrm{T}=4.59 \mathrm{~K}$ to bidirectional pulses (a), positive unidirectional pulses (b), and negative unidirectional pulses (c).

are also present when there is no field and therefore no flux-lines).

A pronounced difference between the response to unidirectional and bidirectional drives is only observed at certain fields, temperatures, and driving currents. The insets of Fig. 2 2 show the current voltage characteristics for unidirectional and bidirectional pulses at $1 \mathrm{KHz}$ for three values of $\mathrm{T}$ at fixed $\mathrm{H}$. The data was obtained with a lock-in voltmeter, which measured the root mean square voltage at the fundamental frequency of the drive. The raw data for the unidirectional drive was multiplied by a factor of 2 to compensate for the fact that the time during which the current is applied is half as long as for the bidirectional case. At $4.55 \mathrm{~K}$, the onset of motion for bidirectional pulses occurs at a threshold current which is a factor of 5 smaller than for the unidirectional case. For the other two temperatures shown, there is little or no difference in the response to the two kinds of drives. The region where there is finite response to bidirectional pulses but no response to unidirectional ones is indicated by the shaded area in the main panel of Fig. 2. Comparison with the DC critical currents shows that a large difference between the unidirectional and bidirectional thresholds only occurs between $T_{m}(H)$ and $T_{p}(H)$.

We next studied the effects of varying the symmetry

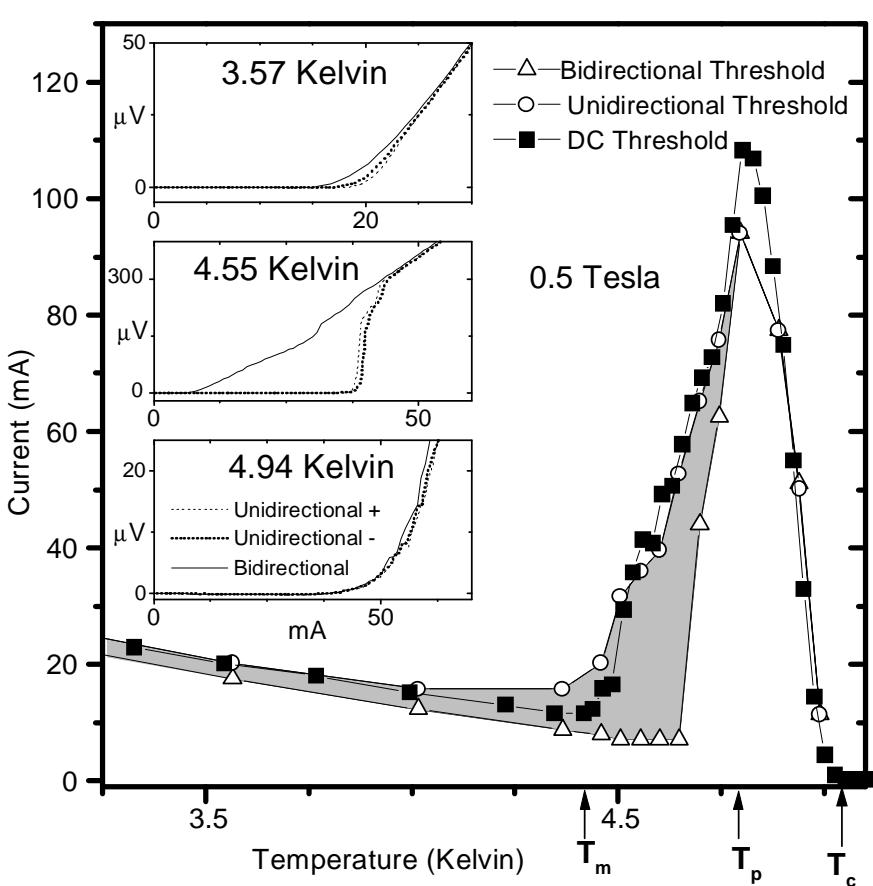

FIG. 2. Main Panel: The temperature dependence of the threshold current at $\mathrm{H}=0.5 \mathrm{~T}$ for unidirectional and bidirectional pulses and for the DC currents. The lines are guides to the eye. The insets show the current-voltage characteristic at three temperatures for each type of pulsed drive.

of the driving current. The response to changing the symmetry of the drive amplitude is shown in Fig. 3ac: an asymmetry in the amplitude (i.e. a larger driving current in one direction than in the other), even when it is small, causes a significant reduction in the response compared with that of the symmetric case. Remarkably, increasing the drive in either direction leads to a sharp decrease in the response (provided the drive is not too large). A similar reduction in the response is also seen if we add a small DC offset to a symmetric sinusoidal driving current. But simply increasing the amplitude of a symmetric bidirectional drive causes the response to increase.

In Fig. Bd-f, the effects of varying the temporal symmetry of the pulse are studied while keeping the pulse amplitude symmetric. The pulse duration in each direction is varied from symmetric (equal pulse lengths) to asymmetric (pulse duration in one direction up to 20 times longer than in the other) while keeping the repeat frequency of pulses fixed. In this case the response is only weakly affected: there is still a substantial voltage when the drive is positive (or negative) $95 \%$ of the time. In this case, there is a net flow of flux-lines, not just back and forth motion. This behavior is reminiscent of the flow of salt in a salt shaker, where the occasional reversal of the force is required to "unjam" the system and maintain the flow. However, this analogy does not work for the amplitude asymmetry effect. 

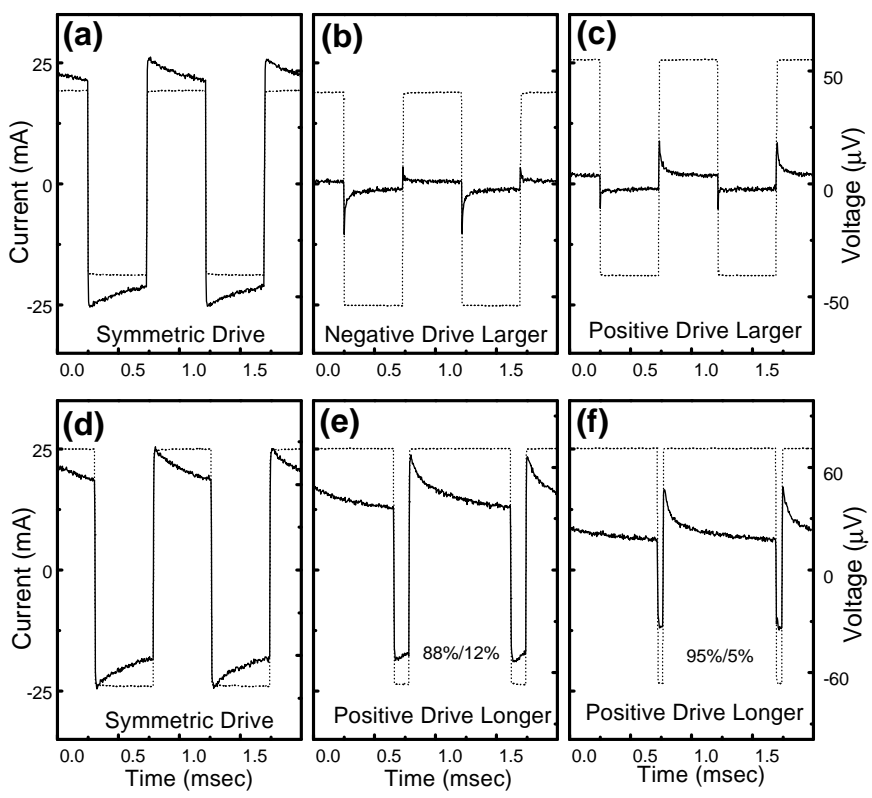

Current (Drive)

Voltage (Response)

FIG. 3. The effect of changing the symmetry of the driving current. In (a) and (d) symmetric bidirectional pulses are applied. In (b) and (c) the amplitude in one direction is slightly larger. In (e) and (f), the amplitude is the same in both directions, but the positive pulses are longer. All data are for $\mathrm{T}=4.59 \mathrm{~K}$ and $\mathrm{H}=0.5 \mathrm{~T}$.

The data shown so far were taken after the response settled to a steady state. If the drive is switched from the bidirectional pulses shown in Fig. 11 a to the unidirectional pulses in $1 \mathrm{~b}$, the response takes some time to decay to zero. We studied such transient effects by observing how the response evolves when the drive is changed. For the data in Fig. : 6 , a $+35 \mathrm{~mA}$ DC current was first applied for several seconds. Subsequently the current was switched back and forth between +35 and $-35 \mathrm{~mA}$ several times, after which it was left at $+35 \mathrm{~mA}$. We see that initially the response is zero, consistent with the fact that $35 \mathrm{~mA}$ is less than the DC critical current. But a small voltage appears as soon as the direction of the current is reversed, and this voltage jumps up to a larger value on each subsequent reversal. If the bidirectional drive persists the response eventually saturates to the steady state shown in Fig. 11a. The voltage decays somewhat between each reversal. After the drive is switched back to DC the decay continues until the response goes back to zero. The entire pattern shown in the figure repeats exactly, if the current is cycled repeatedly through the sequence: DC, bidirectional pulses, DC.

It is important to note that if the current is set to zero after the flux-lines are shaken loose with bidirectional pulses, they remain in an "easy to move" state and give a large response as soon as the current is turned back on. (Waiting times of up to one hour were used.) However, when the current is shut off after applying a DC

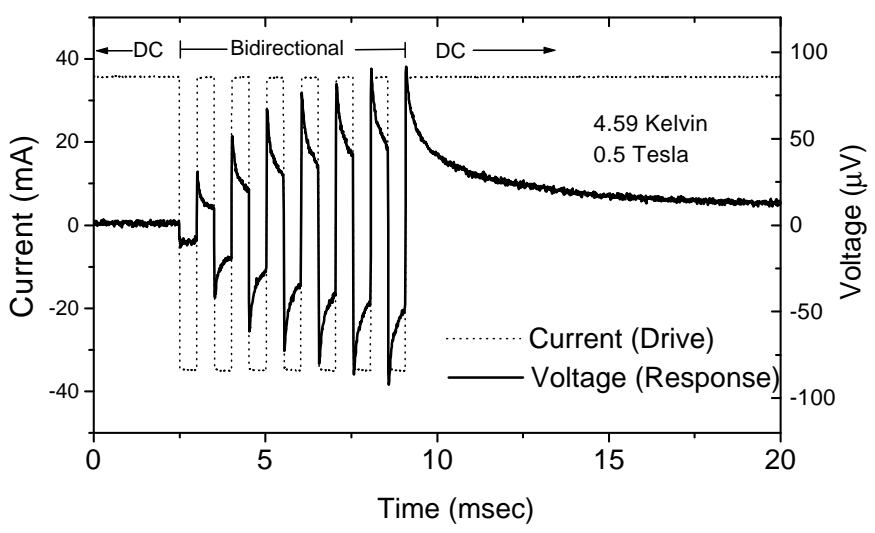

FIG. 4. In this measurement a DC current was first applied for several seconds. Then several bidirectional pulses were applied after which the current was set back to DC.

current, the flux-lines remain in a "hard to move" state and the transient effect shown in Fig. A will occur if bidirectional pulses are subsequently applied. The fact that the FLL "remembers" how it was driven implies that the bidirectional and DC drives cause distinct changes in the structure of the flux lattice. Interestingly, the manner in which the response decays after the drive is switched from $\mathrm{AC}$ to $\mathrm{DC}$, depends on the frequency of the drive, indicating that the frequency plays an important role in determining the FLL structure.

We also studied the frequency dependence of the response for symmetric bidirectional drives (both sinusoidal and square wave), in the range $3 \mathrm{~Hz}-100 \mathrm{kHz}$. In the region of the phase diagram bracketed by $T_{m}(H)$ and $T_{p}(H)$, for driving amplitudes between the unidirectional and bidirectional thresholds, the response is strongly frequency dependent. The in-phase response exhibits a crossover between a low frequency regime in which the response is vanishingly small and a high frequency regime in which the response is a large fraction of the free flux flow value. At the same time the out-of-phase response is negligibly small [17]. The characteristic crossover frequency is in the range $\sim 1-10 \mathrm{kHz}$ and varies with driving amplitude. One could attempt to attribute the crossover to the motion of weakly pinned flux-lines elastically coupled to more strongly pinned ones. However, in this case there would also be a peak (of comparable size to the in-phase response at the crossover frequency) in the frequency dependence of the out-of-phase response which would be directly related to the storage and release of elastic energy during each cycle 18. The fact that the out-of-phase response is negligibly small for the experiments discussed here, strongly suggests that the motion is plastic. The phenomena described here occur for relatively large currents $\left(\geq .2 I_{c}\right)$ only, where the response is highly nonlinear. By contrast, when very small driving currents are used, $I \ll I_{c}$, previous experiments have shown [19] that the system is linear and that it exhibits 
a crossover in the in-phase response together with the large peak in the out-of-phase response at a characteristic "pinning frequency", $\omega_{p} \sim 1-100 \mathrm{MHz}$. This behavior is a direct result of the interplay between the flux flow viscosity and elastic coupling between flux-lines and pinning centers.

The results shown above closely resemble plasticity effects which occur in ordinary solids. When stresses greater than the "yield point" are applied to a solid, regions of the material slide past one another, creating permanent plastic deformations. As plastic flow proceeds in an initially soft material (e.g. annealed copper), becomes progressively more difficult to deform and the motion eventually stops (provided the applied stress is not too large). This phenomenon is known as "strain hardening" 20. The material remains in a hardened state after the applied stress is removed. However, the yield point after strain hardening is typically somewhat anisotropic, i.e. less stress is required to cause plastic flow in the direction opposite to which the object was deformed during the hardening process. When the stress on a heavily strain hardened material is repeatedly reversed, the material becomes progressively easier to deform: this process is known as "cyclic softening" [20]. Strain hardening is usually associated with an increase in the density of dislocations, whereas cyclic softening involves healing of dislocations and reordering of the lattice.

The behavior of the flux lattice can be interpreted in terms of these plasticity effects. As noted above, there is mounting evidence that plastic flow in the FLL involves the formation of channels in which the FLL is more ordered and therefore more weakly pinned than in the surrounding areas. Thus, our results suggest that symmetric back-and-forth shaking of flux-lines facillitates the formation and growth of these channels. However, when a unidirectional drive is applied, the response diminishes quickly, indicating that the easy-flow channels become blocked. This decay of the response resembless the phenomenon of strain hardening in ordinary solids. Since the response is small the first time the direction of the current is reversed after a DC current is applied (Fig. 4), the strain hardening of the FLL must be weakly anisotropic. This is consistent with the observed strong sensitivity to the symmetry of the driving amplitudes (Fig. 3). When the amplitude of the drive is not the same in both directions, the larger amplitude pulses lead to jamming of the channels which cannot be completely unjammed by the smaller amplitude reverse drive.

In summary, we have found that in a well-defined range of fields, temperatures, and driving amplitudes, the flux lattice displays novel types of non-linear response. In particular, the finite frequency current-voltage characteristics exhibit threshold currents that are much smaller than the DC thresholds. The finite frequency response is extremely sensitive to asymmetry in the driving amplitude. However, the response is insensitive to temporal asym- metry in the drive. The memory effects associated with these results show that dynamically generated changes in the structure of the flux lattice are involved. As is the case with ordinary solids, it appears that unidirectional drives tend to disorder the system, while shaking tends to order it. More work is needed, both theoretical and experimental, in order to understand the mechanism leading to these unusual effects of jamming, softening and plasticity and how they are related to the phase diagram of the flux lattice.

Acknowledgments: We thank S. Alexander, S. Cheong, D. Geshkenbein, M. Stephen, R. Stinchcomb, R. Walsted, G. Weng and Z. Xiao for useful discussions; M. Higgins, S. Bhattacharya, M. Oledzka, J. Sunstrom and M. Greenblatt for samples. Work supported by NSFDMR-9705389

$\dagger$ Current Address: Department of Physics, University of California, Los Angeles, California 90024-1547

[1] A. E. Koshelev and V. M. Vinokur, Phys. Rev. Lett. 73 3580 (1994)

[2] M.C. Faleski, M.C. Marchetti and A.A. Middleton, Phys. Rev. B 54, 12427 (1996).

[3] T. Giamarchi and P. Le Doussal, Phys. Rev. Lett. 76 3408 (1996)

[4] S. Bhattacharya, and M. J. Higgins, Phys. Rev. Lett. 70, 2617 (1993); Physica C 257, 232 (1996)

[5] M. C. Hellerqvist, et al. Phys. Rev. Lett. 76, 4022 (1996)

[6] For an early study of plasticity of flux-line lattices see: J. Good and E. Kramer,Phil. Mag. 51, 339 (1971)

[7] U. Yaron et al., Phys. Rev. Lett. 73, 2748 (1994); Nature 376, 753 (1995)

[8] W. Henderson, E.Y. Andrei, M. J. Higgins and S. Bhattacharya, Phys. Rev. Lett. 77, 2077 (1996)

[9] The results described here are qualitatively different from those reported by Gordeev et al. 10]in YBCO.

[10] S.W. Gordeev et. al. Nature 385, 324 (1997)

[11] Wördenweber, R., Kes, P. H. \& Tsuei, C. C. Phys. Rev. B 33, 3172 (1986) Kes, P. H. \& Tsuei, C. C. Phys. Rev. B 28, 5126 (1983)

[12] A. C. Marley, M. J. Higgins \& S.Bhattacharya, Phys. Rev. Lett. 74, 3029 (1995)

[13] H. J. Jensen, A. Brass and A.J. Berlinsky, Phys. Rev. Lett. 60, 1676 (1988)

[14] N. Grønbech-Jensen, A.R. Bishop and D. Domínguez, Phys. Rev. Lett. 76, 2985 (1996)

[15] Reichhardt, C. et al. Phys. Rev. B 53, R8898 (1996)

[16] C.J. Olson, C. Reichardt and F. Nori,to appear in Phys. Rev. Lett.

[17] This follows directly from Fig. 11where the response is seen to be in phase with the drive. The in-phase and out-of-phase response were also measured with lock-in detection.

[18] In any linear system, the Kramers-Kronig relations imply 
that a peak in the frequency dependence of the out-ofphase response must occur when there is a crossover in the frequency dependence of the in-phase response.

[19] W. Henderson, E. Y. Andrei, M.J. Higgins and S. Bhattacharya, Phys. Rev. Lett. 80, 381 (1998).

[20] R. W. Hertzberg, Deformation and Fracture Mechanics of Engineering Materials (John Wiley \& Sons, New York, 1989) ; B. I Sandor, Fundamentals of Cyclic Stress and Strain (Univ. of Wisconsin Press, Madison, 1972) 